International Journal of Current Advanced Research

ISSN: O: 2319-6475, ISSN: P: 2319 - 6505, Impact Factor: SJIF: 5.995

Available Online at www.journalijcar.org

Volume 6; Issue 5; May 2017; Page No. 3763-3767

DOI: http://dx.doi.org/10.24327/ijcar.2017.3767.0364

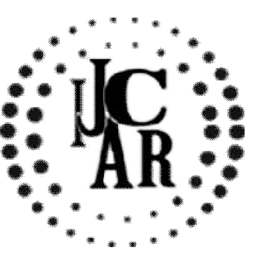

Research Article

\title{
CORRELATION OF CYTOKERATIN 19 EXPRESSIONS TO HISTOLOGICAL GRADING OF ORAL SUBMUCOUS FIBROSIS: AN IMMUNOHISTOCHEMICAL STUDY
}

\author{
Shan Nawaz Malik ${ }^{*}$,. Zankana Vyas ${ }^{2}$., Hemanth Kotari ${ }^{3}$., \\ Vishnudas Dinesh Prabhu ${ }^{4}$ and Mariyam Shahina k E ${ }^{5}$ \\ 1,2,3Pacific university, Udaipur Rajasthan, India \\ ${ }^{4}$ Department of Oral and Maxillofacial Pathology Yenepoya dental college, \\ Yenepoya University Mangalore, India \\ ${ }^{5}$ St Johns Medical College Bangalore, Karnataka, India
}

\section{A R T I C L E I N F O}

\section{Article History:}

Received $20^{\text {th }}$ February, 2017

Received in revised form $12^{\text {th }}$ March, 2017

Accepted $15^{\text {th }}$ April, 2017

Published online $28^{\text {th }}$ May, 2017

\section{Key words:}

Immunohistochemistry (IHC), OSF(Oral submucous fibrosis, OSCC(Oral squamous cell carcinoma), NOM(normal oral mucosa).

\begin{abstract}
A B S T R A C T
Introduction: Immunohistochemical studies have become one of the stepping stones to establish new surrogate markers to analyse the malignant transformation of a potentially malignant disorder to malignancy

Objective: The histological grading of oral submucous fibrosis has been made as grade 1,2 and 3 according to severity of fibrosis and hyalinization but the intensity of immunohistochemical staining of cytokeratin 19 has not been done to analyse and establish the fact whether the intensity increases according to histologic severity.
\end{abstract}

\begin{abstract}
Material and methods: 35 cases of OSF were histologically graded and immunohistochemical study was done using cytokeratin 19.The $\mathrm{P}$ value were calculated using Fisher's Exact tests.

Results: Among 35 cases 11 cases of grade 1 showed $18.1 \%$ of negetive, $36.3 \%$ of mild, $36.3 \%$ of moderate and $9 \%$ of intense staining. 9 cases of Grade 2 showed $11.1 \%$ of negative, $33.3 \%$ of mild, $33.3 \%$ of moderate and $22.2 \%$ of intense staining. 15 cases of Grade 3 showed $13.3 \%$ negative, $26.6 \%$ mild, $40 \%$ moderate and $20 \%$ intense staining. The overall and comparative $\mathrm{P}$ values were insignificant.
\end{abstract}

Conclusion: Cytokeratin 19 staining alone cannot be used as a surrogate marker to check the severity and transformation of OSF to OSCC in different histologic grades of OSF.

Copyright $₫ 2017$ Shan Nawaz Malik et al. This is an open access article distributed under the Creative Commons Attribution License, which permits unrestricted use, distribution, and reproduction in any medium, provided the original work is properly cited.

\section{INTRODUCTION}

Potentially malignant disorders, previously called as premalignant condition are occurring quite frequently in general population in Asian countries and OSF is one of them. The study provides a detailed interaction between OSF and CK19 based on the histological grades of OSF.

Recently, various Immunohistochemical markers are showing great promise in helping to predict prognosis and response therapy, even at a very early point of tumor development or may it be a premalignant condition like $\mathrm{OSF}^{1}$. Alteration of CK19 expression has been documented in leukoplakia and oral cancer ${ }^{2}$. The present study is carried out to characterize the CK19 profile in OSF based on the histological grades of OSF and ascertain if this could be used as a surrogate marker

*Corresponding author: Shan Nawaz Malik Pacific university, Udaipur Rajasthan, India for malignant transformation and if the results hold positive then CK19 can be the ideal surrogate marker that can be used to analyze and characterize the rate and conversion of OSF to OSCC or any malignant lesion correlating it with histological grading of OSF.

\section{MATERIALS AND METHODS}

\section{Hypotheses To Be Tested}

Whether CK19 can be used as a surrogate marker for malignant transformation of OSF based on whether the intensity increases according to histologic severity.

\section{Source Of Data}

Total of 35 cases of OSF cases were taken for the study.

Confirmed OSF cases were taken for this study and were collected from Oral Pathology department archives Yenepoya Dental College, Mangalore, Karnataka, India. All ethical approval concerned to the study was taken from Yenepoya dental college ethical committee. 
They were histologically divided based on fibrosis and hyalinization into

Grade 1:

Loose, thick and thin fibers

Grade II:

Loose or thick fibers with partial hyalinization.

Grade III:

Complete hyalinization $^{3}$

\section{Sample Size}

Inclusion criteria: 35 cases of histologically confirmed cases of OSF were included Exclusion criteria: Sections of size less than $2 \mu \mathrm{m}$ were excluded.

\section{Collection Of Data}

Sections of $3 \mu \mathrm{m}$ thickness were prepared from the formalin fixed, paraffin embedded tissue blocks. The sections were mounted on poly L-lysine coated slides for CK 19 expression the staining was done immunohistochemically using polymerase technique, the primary and secondary antibody for the study was obtained from Biogenex, Bangalore.

\section{Tools And Techniques Of Research}

Before getting started with IHC study two types of buffer were prepared.

1. Tris buffer

2. Cirate buffer

These were prepared by the following method

\section{Tris Buffer}

First 1N HCL was prepared which is used to make both the solutions.1N HCL was prepared by adding conc-HCL slowly drop by drop to $91 \mathrm{ml}$ of distilled water in an open jar with dissolving motion so that it gets well dissolved. Tris buffer was prepared by initially adding $0.605 \mathrm{Gms}$ of tris buffer powder and eight Gms of sodium chloride powder to $1,000 \mathrm{ml}$ of distilled water and dissolve it, later four ml of $1 \mathrm{~N}$ HCL was added drop by drop with shaking to the tris solution to bring the $\mathrm{pH}$ to 7.6. The $\mathrm{pH}$ of the solution was measured by using $\mathrm{pH}$ meter strip.

\section{Cirate buffer}

( Retrieval buffer) it was prepared by adding $2.9 \mathrm{Gms}$ Trisodium citrate to $1,000 \mathrm{ml}$ of distilled water, 1N HCL (five $\mathrm{ml}$ ) was added slowly drop by drop to this citrate solution to bring it to $\mathrm{pH}$ of around six to 6.2 . The $\mathrm{pH}$ was measured by using $\mathrm{pH}$ meter strip. The tris and the citrate buffer were stored in refrigeration for a period of one week for reuse. Antigen retrieval was done by initially deparaffinizing the section in xylene for 30 minutes then the slides were kept in absolute alcohol for two mins in a couplin jar then the slides were again kept in absolute alcohol for two mins in another couplin jar, then rinsed in water for five to ten mins and then rinsed in distilled water.

The slides were placed in cooker with citrate buffer and heated for two to three whistles, cooker were then placed under tap water for cooling to room temperature. Slides after cooling were rinsed in water then rinsed in distilled water (couplin jar) then immersed in Tris buffer for five mins (couplin jar)

\section{IHC Procedure}

The moisture chamber was prepared by keeping a wet filter paper in a steel tray with its lid (cover). One drop of peroxide block was placed on the section and the slides were placed in the moisture chamber for ten mins. The slides were then rinsed in tris buffer (couplin) for five minutes with two changes. Then the sections were covered with power block and left for ten minutes in the moisture chamber, washing was not needed after this procedure, excess was just wiped with tissue paper and then the sections was covered with primary antibody and left in moisture chamber for one hour.

The slide was then rinsed for five minutes in Tris buffer with two changes, sections were then covered with one drop of super enhancer and kept in the moisture chamber for 30 minutes and then rinsed for five minutes in Tris buffer with two changes. The sections were then covered with one drop of SS label and kept in moisture chamber for 30 minutes. The coloring material chromogen were prepared by adding 1 drop of liquid DAB chromogen \& $1 \mathrm{ml}$ stable DAB buffer in a mixing vial and mixed well, sections were rinsed in Tris buffer for five minutes with two changes then the section were stained for five-eight minutes with the above mixture of chromogen keeping it inside the moisture chamber. Then it was dipped in tris buffer in couplin jar and rinsed in running water for five minutes, counter staining was done in hematoxylin for $1 \mathrm{~min}$, then washed, dried and treated with few drops of xylene for clearing \& mounted it on DPX immediately.

Table 1 Histological staging of severity of OSF Pvalue $=0.136$

\begin{tabular}{ccccccc}
\hline Lesions/control & \multicolumn{4}{c}{ CK-19 EXPRESSION } & \multirow{2}{*}{ Total } \\
\cline { 2 - 5 } & negative & Mild & moderate & Intense & \\
\hline OSFgrade 1 & 2 & 4 & 4 & 1 & $11(100 \%)$ \\
Osf grade 2 & 1 & 3 & 3 & 2 & $9(100 \%)$ \\
Osf grade 3 & 2 & 4 & 6 & 3 & $15(100 \%)$ \\
Total & 5 & 11 & 13 & 6 & $35(100 \%)$ \\
\hline
\end{tabular}

All the sections were coded before staining for CK19, Evaluation of CK19 was done under light microscope under $10 \times$ objective and the intensity of staining of epithelium was assessed as (-) negative, (+) mild, (++) moderate, $(+++)$ intense, the sections were decoded and results tabulated ${ }^{1}$.

\section{The intensity of staining was analyzed by the percentage of tissue section stained per slide.}

If no tissue is stained - Negative

If $1 / 3$ of the epithelium tissue is stained (approximately $33 \%)$ - Mild

If $2 / 3$ of the epithelium tissue is stained (approximately $66 \%)$ - Moderate

If more than $2 / 3$ of the epithelium tissue is stained (above $66 \%$ ) -Intense ${ }^{1}$.

Two independent observers evaluated the slides when discrepancy existed a third pathologist was asked to evaluate the slide to arrive at the consensus conclusion, 35 cases of OSF section were analysed after dividing them based on 
grade as grade1,2 and 3 respectively. A total of 35 sections altogether were analysed.

\section{Statistical Analysis}

The results were calculated using Fisher's Exact test. All statistical analyses were done using STATA 14. P value less than 0.05 was considered statistically significant. Photomicrographs were obtained using CX-(Olympus) microscope

\section{RESULTS}

Out of 35 cases of OSF, 11 cases were grade1, 9 cases were grade 2 and 15 cases were grade 3. CK19 expressions in all three grades are shown in thetable1, supported by figure1, figure 2 , figure 3 and figure 4 .

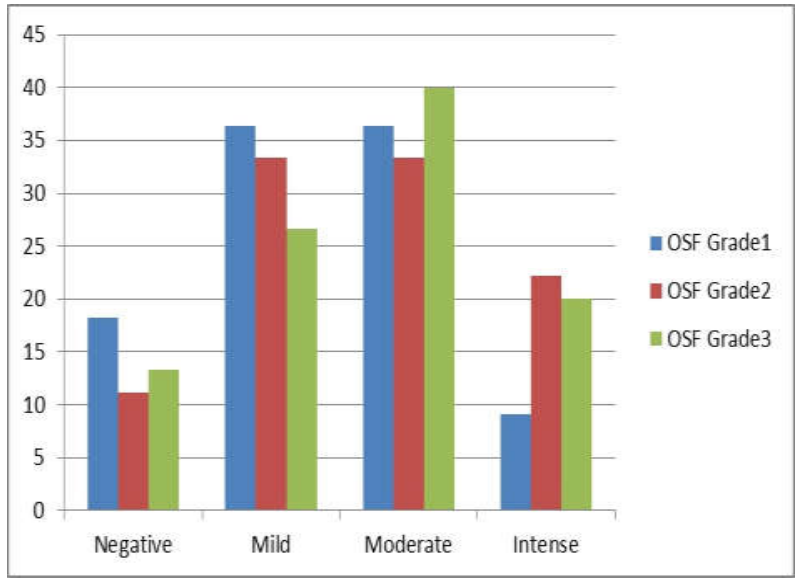

Figure 1 Instensity Of Staining Of Ck19

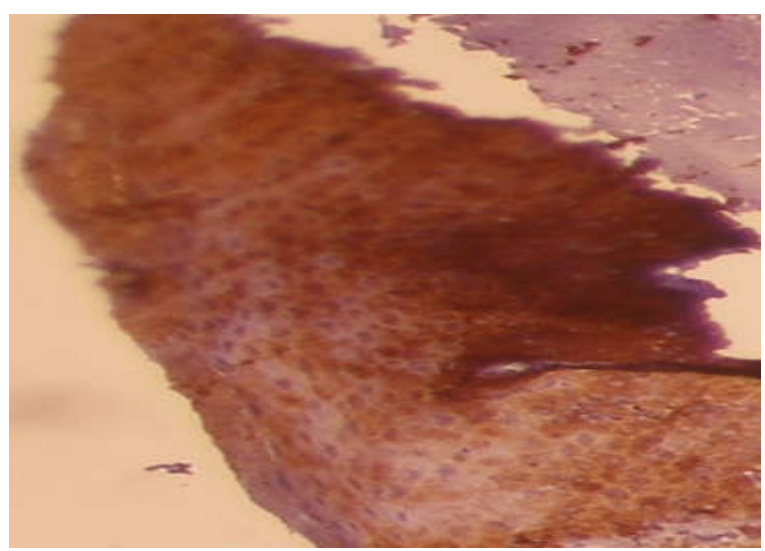

Figure 2 Osf Grade 1 Ck19 Staining (10 X Magnifications)

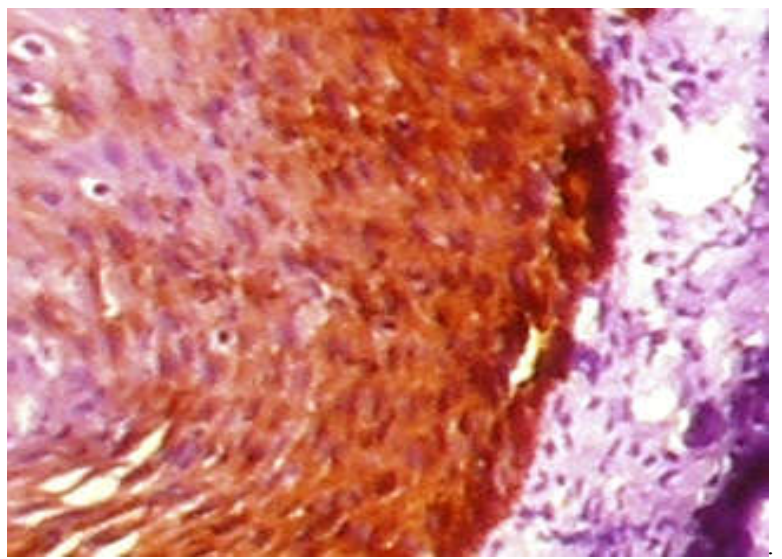

Figure 3 Osf Grade 2 Ck19 Staining (10 X Magnifications)

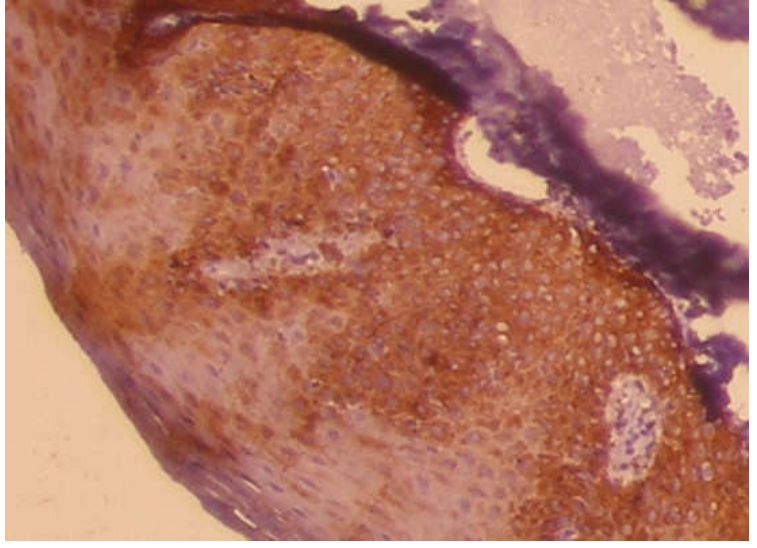

Figure 4 Osf Grade 3 Ck19 Staining (10 X Magnifications)

After Fisher's Exact test The P value $=0.995 \mathrm{~ns}$. Since the total $\mathrm{P}$ value is $0.136 \mathrm{~ns}$ and

$\begin{array}{ll}\text { Grade 1 vs grade 2 } & 1.000 \\ \text { Grade 1 vs grade 3 } & 1.000 \\ \text { Grade 2 vs grade 3 } & 0.945\end{array}$

There is no significant difference between the intensity levels and were considered statistically insignificant.

\section{DISCUSSION}

Oral potentially malignant disorder (OPMD) is a common term suggested to replace oral pre-cancer, Including both oral precancerous lesions and oral Precancerous conditions. All oral lesions that carry a risk of malignant transformation are included under this term. OSF in of the oral potentially malignant disorders ${ }^{4}$.

OSF is a chronic, progressive scarring disease, which predominantly affects the people of south- East Asian origin ${ }^{5}$. The early signs and symptoms of this disorder are intolerance to spicy food, altered salivation, ulceration, vesiculation, pigmentation changes, recurrent stomatitis and petechiaes. Extra orally, there may be loss of pigmentation or excessive pigmentation of vermilion borders in some of the cases ${ }^{6}$. The buccal mucosa, retro molar area and soft palate are the most commonly affected sites. The mucosa in this region develops a blotchy, marble - like pallor and a progressive stiffness of sub epithelial tissues ${ }^{7}$.

Clinically OSF cases can be categorized into three clinical stages according to their ability to open the mouth, as given below.

Stage I - Mouth opening $\geq 45 \mathrm{~mm}$

Stage II - Restricted mouth opening 20-44mm

Stage III - mouth opening $\leq 20 \mathrm{~mm}^{8}$

A vivo study on mouse model for study of development of OSF by Sumetha Perera (2007) showed the areca- nut treated oral epithelium showed progressive changes in epithelial thickness leading to atrophy, increased cellularity of fibroblasts, fibrosis of connective tissue, focal infiltration of inflammatory cells and muscle atrophy ${ }^{9}$ Histologically OSF can be grouped into four clearly definable stages: very early, early, moderately advanced and advanced. These stages are based on nature of the sub epithelial collagen, presence or absence of edema, physical state of the mucosal collagen, overall fibroblastic response state of blood vessels and 
predominant cell type in the inflammatory exudate. The connective tissue in advanced state is characterized by the sub mucosal deposition of extremely dense and a vascular collagenous tissue with variable numbers of chronic inflammatory cells ${ }^{4}$. Kiran Kumar (2007) proposed histological grading as follows:

Grade 1:Loose, thick and thin fibers

Grade II: Loose or thick fibers with partial hyalinization.

Grade III: Complete hyalinization ${ }^{10}$

All epithelial tissues have cytokeratin in them and potentially malignant disorders like OSF have epithelial component.

Cytokeratin's that form the cytoskeletons of the epithelial cells are of several molecular types. The patterns of expression of these different types of CKs vary depending upon the type of epithelial cells and hence, they may be used as potential markers of cell differentiation and malignant transformation. CK 19, a type I (acidic) keratin, is the smallest keratin and is unique in that it lacks the Carboxyterminal, non- $\alpha$ - helical tail domain, which is typical for all other keratins ${ }^{11}$.

In a study done by Ali Yousif (2014) Expressional profile of both markers (p16 and CK19) was different in same tumour. CK19 positivity was associated with age whereas p16 showed insignificant expression. The expression of p16 decreased while CK19 increased with the tumour grade. This finding also shows that progressive loss of 16 and high expression of CK19 according to tumor metastasis. When p16 and CK19 evaluated together may have more accurate prediction of clinical outcome/prognostic marker in human OSCC and important molecular event in pathogenesis of oral carcinoma ${ }^{12}$ Anand Lalli (2008), in his IHC study observed an increase of $\mathrm{KI}$ and $\mathrm{K} 10$ in the suprabasal layer, induction of K6 in the basal layer and completes loss of K19 in theepithelium. In a subset of the most severe OSF cases (14\%), K17 expression was completely lost in the basal layer which might define them to be at most risk to undergo malignant transformation. There was no detectable expression of K8, K18, K7 and K9 and the expression of K4, K13, K14, K15 and K16 did not change in $\mathrm{OSF}^{13}$.

In an IHC study by W.M. Tilakaratne (2008), the data indicated that (hypoxia inducible factor) HIF-1 $\alpha$ is up regulated at both protein and mRNA levels in OSF and the correlation with dysplasia is statistically significant. HIF $-1 \alpha$ may play a role in malignant transformation of OSF. Further over expression of HIF $-1 \alpha$ may contribute to the progression of fibrosis. It may be possible to use HIF $-1 \alpha$ as a marker for malignant transformation of $\mathrm{OSF}^{14}$ Unlike our study, an IHC study by K. Ranganathan (2006), Significant difference in the CK staining pattern was seen between normal, OSF and OSCC. Significant changes in OSF included increased intensity of staining for (Pan Cytokeratin) panCK and (high molecular weight cytokeratin) HMWCk, aberrant expression of CK8 and decreased expression of CKs 5 and $14^{2}$.

Our study which was limited to histological grades of OSF, showed a total of 5 negative, 11 mild, 13 moderate and 6 intense staining out of 35 cases. Among 35 cases 11 cases of grade 1 showed $18.1 \%$ of negative, $36.3 \%$ of mild, $36.3 \%$ of moderate and $9 \%$ of intense staining. 9 cases of Grade 2 showed $11.1 \%$ of negative, $33.3 \%$ of mild, $33.3 \%$ of moderate and $22.2 \%$ of intense staining. 15 cases of Grade 3 showed $13.3 \%$ negative, $26.6 \%$ mild, $40 \%$ moderate and $20 \%$ intense staining Our study also observed that there is always high level of staining with CK 19 which also correlated with other cytokeratin studies ${ }^{1,2,12}$ that show good staining concluding the act that cytokeratin's have strong staining potential compared to any other proteins.

\section{CONCLUSION}

Though few studies have shown that pancytokeratins can be used as a surrogate marker, In our study, there was no significant difference between the intensity levels of OSF among 3 histological grades, hence, this study showed that CK19 profile alone cannot be used to ascertain if it could be used as a surrogate marker for malignant transformation of OSF. Hence our study says that that CK19 does not play a significant role alone in the transformation of OSF TO OSCC. More IHC studies have to be done on different cytokeratin individually to explore a wider diagnostic usage in future.

\section{Acknowledgment}

We would like to sincerely acknowledge all the non-authors for their technical support for the present study

\section{References}

1. Malik S N, Alam M K.2014.Cytokeratin-14 Expression in Normal Oral Mucosal Tissue, Oral Submucos Fibrosis and Oral Squamous Cell Carcinoma: An Immunohistochemical Study. International Medical Journal., 21: 223 - 225.

2. Ranganathan K, Kavitha R.2006.Cytokeratin expression in oral submucos fibrosis- an Immunohistochemical study. $J$ oral pathol med., 35:25-32.

3. Kumar K, Saraswathi TR .2007. Oral sub mucous Fibrosis: A clino-histopathological study in Chennai. Indian journal of dental research., 18:106-111.

4. Prabhakaran S P, Muthukrishnan A.2014. Expression of cytokeratin 18 and 19 in potentially malignant disorders: a systemic review, Journal of Indian academy oral medicine and radiology., 26:173-177.

5. Shafer WG, Hine MK.2007.A Textbook of Oral Pathology Fifth edition: Saunders., 136-141.

6. Saraf S, Gandal R.2006. Textbook of oral pathology First edition: Jaypee Medical Publishers., 242-246.

7. Neville BW, Damm DD.1995. Oral \& Maxillofacial Pathology: Saunders: 291.

8. Kumar K, Saraswathi TR.2007. Oral sub mucous Fibrosis: A clino-histopathological study in Chennai. Indian Journal of Dental Research., 18:106-111.

9. Perera MWS, Gun singhe D.2007. Development of an in vivo mouse model to study oral submucos fibrosis. Oral pathol med., 36: 273-80.

10. Kumar Kiran. Sarasvvathi TR, Rangnathan K. Devi Lima M, Elizabeth Joshua.2007. Oral submucous fibrosis: A clinico- histopathological study in Chennai. Indian Journal of Denial Research., 18(3): 106-11.

11. Kamath K P, vidya M.2015. Cytokeratin 19 expression patterns of dentigerous cysts and 
odontogenic keratocysts, Annals of medical and health sciences research., 5:119-123.

12. Ali Yousif babiker, Arshad A rehmani.2014.Expression analysis of p16 and cytokeratin 19 protein in the genesis of oral squamous cell carcinoma patients. Int $J$ Clin Exp Med., 7(6)1524-1530.
13. Lalli A, Tilakaratne WM.2008.An altered keratinocyte phenotype in oral sub mucous fibrosis; correlation of keratin K17 expression with disease severity. J of oral pathol med., 37:211-220.

14. Tilakaratne WM, Iqbal Z.2008.Up regulation of HIF$1 \alpha$ in malignant transformation of oral sub mucous fibrosis. J of oral pathol med., 37:372-377.

\section{How to cite this article:}

Shan Nawaz Malik et al (2017) 'Correlation Of Cytokeratin 19 Expressions To Histological Grading Of Oral Submucous Fibrosis: An Immunohistochemical Study, International Journal of Current Advanced Research, 06(05), pp. 3763-3767. DOI: http://dx.doi.org/10.24327/ijcar.2017.3767.0364 